> La tyrosine kinase ZAP-70 (zeta-chain-associated protein of $70 \mathrm{kDa}$ ) a connu son heure de gloire au début des années 1990, lorsque des mutations du gène codant pour cette protéine ont été mises en évidence chez de très jeunes patients présentant des déficits immunitaires combinés sévères. La caractérisation, chez ces patients, de déficits fonctionnels des lymphocytes $T$ signait le rôle crucial de cette protéine dans l'activation de ces cellules. Très récemment, un regain d'intérêt pour ZAP-70 est apparu lorsque son expression, dans un premier temps considérée comme spécifique des lymphocytes $T$ et des cellules NK (natural killer), a été mise en évidence dans certaines leucémies lymphoïdes chroniques $B$ et associée à un facteur pronostique. Enfin, la découverte d'une mutation ponctuelle spontanée de ZAP-70 dans une souche de souris présentant une arthrite rhumatoïde révélait également le rôle potentiel de ZAP-70 dans des maladies auto-immunes. Les diverses maladies associées à des modifications d'expression ou d'activité de ZAP-70 démontrent le rôle clé de cette kinase dans la mise en place et la régulation d'une réponse immunitaire. <

\section{Tout ce que vous avez toujours voulu savoir sur la protéine ZAP-70}

\section{Claire Hivroz}

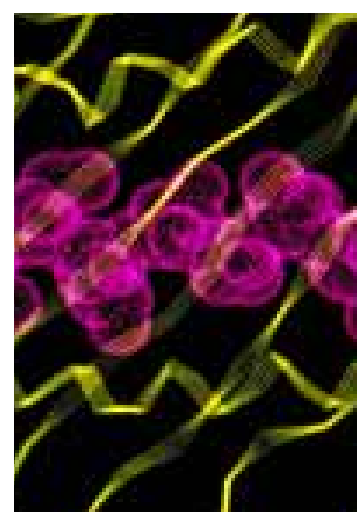

Inserm U.365, Institut Curie,

Section Recherche,

26, rue d'UIm,

reconnaissance de 75248 Paris Cedex 05, France. leurs ligands. Quatre claire.hivroz@curie.fr familles de protéine tyrosine kinases sont impliquées dans la mise en place de la réponse $T$ : les kinases des familles Src, Tec, Csk et les kinases de la famille Syk/ZAP-70. Le rôle clé de la famille Syk/ZAP-70 a été mis en évidence au début des années 1990 grâce à la découverte de patients présentant des déficits immunitaires combinés sévères dus à des mutations du gène zap-70. Depuis, cette protéine tyrosine kinase n'a cessé d'intéresser les immunologistes. Nous présenterons ici le rôle de ZAP-70 dans l'activation des lymphocytes $T$ et $B$, en insistant sur ce que les maladies associées à des modifications d'expression et d'activité de ZAP-70 nous apprennent sur la fonction de cette kinase.

\section{La protéine ZAP-70}

peptide antigénique présenté par les molécules du complexe majeur d'histocompatibilité pour le TCR, un antigène soluble pour le $B C R$ ) et des chaînes impliquées dans la transduction du signal. La reconnaissance de ces ligands induit la phosphorylation sur des résidus tyrosine de nombreuses protéines, préambule obligatoire aux réponses fonctionnelles, quelles qu'elles soient: prolifération, différenciation, fonctions effectrices.

$T C R$ et $B C R$ n'ont pas d'activité kinase intrinsèque, mais recrutent des protéine tyrosine kinases intracellulaires qui s'associent transitoirement aux récepteurs après
La stimulation des lymphocytes T par le TCR induit la phosphorylation sur des résidus tyrosine de nombreuses protéines, dont les chaînes CD3 et $\zeta$ associées au TCR. Ces chaînes contiennent des motifs intracytoplasmiques, nommés immunoreceptor tyrosine-based activation motifs (ITAM), contenant chacun deux résidus tyrosine phosphorylés par des protéine kinases de la famille Src. En 1992, le groupe de A. Weiss mettait en évidence une activité tyrosine kinase, d'un poids moléculaire apparent 
de 70 kDa, associée à la chaîne $\zeta$. Le clonage du gène codant pour cette protéine révélait l'existence d'une nouvelle protéine tyrosine kinase appelée $\zeta$ associated protein of $70 \mathrm{kDa}$, soit ZAP-70 [1]. Cette kinase présentait des caractéristiques différentes des protéine tyrosine kinases appartenant à la famille Src. ZAP-70, dans les lymphocytes T au repos, est intracytosolique et ne s'associe à la membrane qu'après stimulation du TCR. Cette association s'effectue grâce à la reconnaissance, par deux domaines présents dans ZAP-70 (les domaines SH2) (Figure 1), des motifs ITAM phosphorylés présents dans les chaînes associées au TCR. Une fois recrutée dans la membrane, ZAP-70 est phosphorylée, activée et peut alors phosphoryler ses substrats [2]. Enfin, ZAP-70 est membre d'une famille de kinases qui ne contient pour l'heure que deux membres, Syk et ZAP-70, de structure très similaire.

\section{Déficits immunitaires associés à des mutations de zap-70}

La découverte - peu de temps après le clonage du gène zap-70 - de patients atteints de déficits immunitaires présentant des mutations de ce gène démontra de façon formelle le rôle indispensable de la kinase ZAP-70 dans la réponse immunitaire [3-5].

Les déficits immunitaires liés à des défauts de ZAP-70 sont des déficits dits combinés sévères (DICS), autosomiques récessifs, rares. La grande majorité des patients souffrant de ce DICS présentent très tôt des signes cliniques graves: infections pulmonaires souvent liées à des agents pathogènes opportunistes, diarrhées chroniques, retard de croissance et candidoses persistantes [6]. Cette forme de DICS asso-

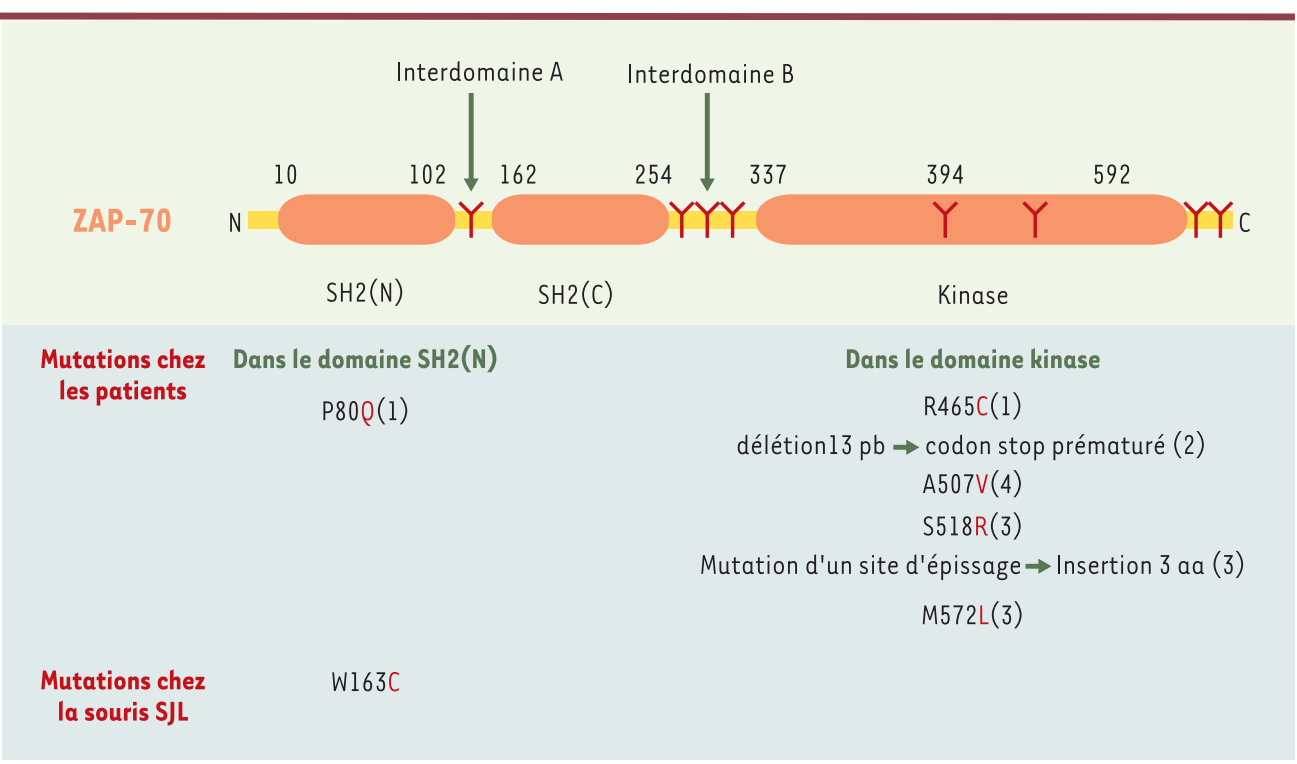

Figure 1. Structure de la tyrosine kinase ZAP-70. ZAP-70 est une kinase intracytosolique qui comporte deux domaines SH2 s'associant à des motifs phosphorylés sur des résidus tyrosine, un domaine kinase et de nombreux résidus tyrosine $(\mathrm{Y})$ dont la phosphorylation règle positivement ou négativement son activité: ainsi, la phosphorylation des tyrosines 394 et 592 règle positivement et négativement, respectivement, l'activité kinase de ZAP-70. Les mutations rapportées chez l'homme sont indiquées, les nombres entre parenthèses indiquant le nombre de patients présentant la mutation. La mutation de ZAP-70 mise en évidence chez la souris SJL est également indiquée (pb: paires de bases; aa: acides aminés) (d'après le site http://www.uta.fi/imt/bioinfo/ZAP70base). ciée à des mutations de zap-70 a souvent une issue fatale. Le seul traitement, à ce jour, est la greffe de moelle osseuse, plusieurs patients ayant ainsi été traités avec succès. Des expériences de ré-introduction, dans des cellules CD4 $4^{+}$de patients, du gène zap-70 grâce à des vecteurs rétroviraux ont montré une reconstitution fonctionnelle avec un avantage sélectif d'expansion pour les cellules T exprimant le transgène [7]. La thérapie génique pourrait donc constituer un traitement alternatif.

Les déficits en ZAP-70 sont caractérisés par une absence de lymphocytes $T \mathrm{CD}^{+}$en périphérie et un nombre normal, ou accru, de lymphocytes $T C D 4^{+}$, qui ne peuvent pas être activés par stimulation du TCR. Cependant, ces critères ne suffisent pas à établir un diagnostic sûr, qui ne peut être fait qu'après mise en évidence de mutations du gène zap-70.

À l'exception d'un cas, toutes les mutations identifiées chez les patients se situent dans une région essentielle à la stabilité et à l'activité de ZAP-70, le domaine kinase (Figure 1). Les mutations décrites à ce jour sont homozygotes ou hétérozygotes, consistent en des insertions, des délétions ou des substitutions, et se traduisent par une absence d'expression de la protéine. Dans un cas, rapporté par un groupe japonais, les mutations hétérozygotes, l'une dans un domaine $\mathrm{SH} 2$, l'autre dans le domaine kinase, se traduisent par une dégradation de la protéine ZAP-70 dépendante de la température [8]. Ce patient, chez qui ZAP-70 n'est pas complètement absente, présente des signes cliniques différents et, notamment, des lésions cutanées liées à une infiltration importante de la peau par des lymphocytes $\mathrm{T} \mathrm{CD}^{+}$, de phénotype mémoire, activés [9]. On peut imaginer que, chez ce patient chez lequel la protéine ZAP-70 est rapidement dégradée à $37^{\circ} \mathrm{C}$, la kinase puisse être exprimée dans les lymphocytes $T$ de la peau, 
où la température est légèrement inférieure; cette expression activerait alors ces lymphocytes T autoréactifs. Nous reviendrons à la fin de cet article sur le rôle que peut jouer ZAP-70 dans la production de lymphocytes T autoréactifs.

\section{Voies de signalisation contrôlées par ZAP-70}

Plusieurs modèles ont permis de mettre en évidence les voies de signalisation contrôlées par la protéine ZAP70: les patients, que nous venons de décrire, un clone dérivé de la lignée T leucémique Jurkat, qui n'exprime ni ZAP-70 ni Syk [10] et, enfin, des souris zap-70-1-, décrites après les déficits humains et obtenues par recombinaison génétique [11]. Des différences entre les déficits observés chez la souris et chez l'humain ont été observées: ainsi, les souris n'ont ni lymphocytes T $\mathrm{CD}^{+}$, ni lymphocytes $\mathrm{T} C D 8^{+}$en périphérie, tandis que seule la population $\mathrm{CD}^{+}$ est absente chez l'homme (Figure 2). Cela rappelle, s'il en est encore besoin, que l'on ne peut pas toujours généraliser à l'homme les résultats obtenus dans des modèles animaux.

\section{À la recherche des} substrats de ZAP-70 L'un des premiers défauts mis en évidence dans les thymocytes ou les lymphocytes $T$ déficients en ZAP-70 est l'absence d'augmentation de la concentration en $\mathrm{Ca}^{2+}$ libre intracellulaire après stimulation du TCR [10]. Un défaut d'activation des MAP-kinases (mitogen-activated proteinkinases, MAPK) par le TCR a également été observé dans les thymocytes de souris $z a p-70^{-/-}$[11] et dans les lymphocytes $T$ $C D 4^{+}$des patients déficients en ZAP-70 [12]. Dans le modèle du clone Jurkat n'exprimant ni

Différenciation T

Différenciation B
ZAP-70, ni Syk, une activation des MAPK par stimulation du TCR a été constatée [13], suggérant que l'activation de cette voie n'est pas entièrement dépendante de ZAP-70.

Une recherche systématique des protéines non phosphorylées dans les lymphocytes T de cellules déficientes en ZAP-70 a mis en évidence des défauts de phosphorylation de deux protéines en particulier: LAT (linker for activation of T cells) et SLP76 (SH2-containing leukocyte protein of $76 \mathrm{kDa})[14,15]$. Ces protéines, dépourvues d'activité enzymatique intrinsèque, contiennent en revanche de nombreux résidus tyrosine qui, une fois phosphorylés, s'associent à d'autres protéines et permettent ainsi l'organisation dans l'espace de molécules de signalisation et l'assemblage de cascades activatrices [16] (Figure 3). Ce contrôle de la phosphorylation de ces molécules d'« échafaudage » par ZAP-70 explique pourquoi ZAP-70 se situe au carrefour de plusieurs voies d'activation.

L'activité kinase de ZAP-70 contrôle également la communication entre cellules du système immunitaire, notamment en réglant l'adhérence des lymphocytes T aux cellules présentatrices d'antigène (CPA) [17]. Cette activité nécessite en effet la phosphorylation de LAT, son asso-
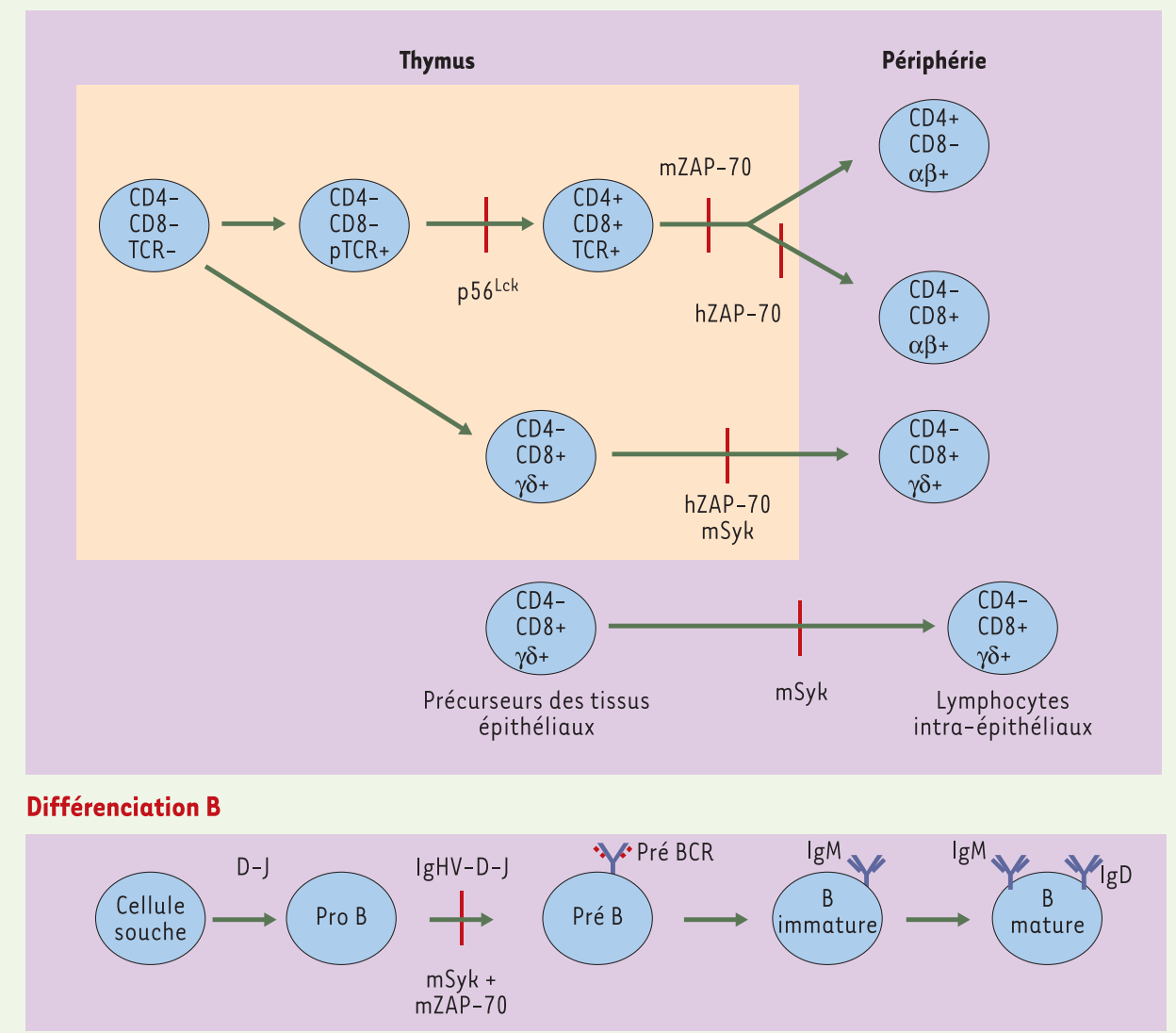

Figure 2. Rôle de Syk et de ZAP-70 dans la différenciation T et B. La différenciation des populations T et B est représentée schématiquement. Les blocages de maturation chez l'homme (h) et chez la souris ( $m$ ) liés à l'absence de ZAP-70 et/ou de Syk (I'autre kinase membre de la famille zap-70) sont représentés par des barres rouges. $p 56^{\text {Lck }}$ : protéine tyrosine kinase activée après stimulation du TCR. 
ciation à SLP76 et à GADS (Grb2-related adapter downstream of Shc) [8] (Figure 3) et, vraisemblablement, des modifications du cytosquelette d'actine. Nous avons également démontré que ZAP-70 contrôle la polarisation des lymphocytes T vers la CPA [18]. Cette polarisation est extrêmement importante puisqu'elle permet la délivrance d'effecteurs (lymphokines ou granules cytolytiques, par exemple) de façon dirigée, évitant ainsi les effets non spécifiques de ces effecteurs sur les cellules environnantes [19]. Ainsi, en contrôlant l'adhérence des lymphocytes T aux CPA et leur polarisation vers la CPA, ZAP-70 assure-t-elle la spécificité d'interaction entre ces cellules et la formation de la zone d'interaction, appelée synapse immune (Figure 4).

\section{ZAP-70 est aussi impliquée dans l'inhibition des lymphocytes T}

La kinase ZAP-70, décrite comme enzyme clé de l'activation des lymphocytes $T$, joue également un rôle dans la mise en place de mécanismes inhibiteurs de la réponse immune.

Le TCR est progressivement internalisé et dégradé après avoir reconnu son ligand. Cette dégradation entraîne la fin progressive de la réponse T par disparition du TCR de la surface des lymphocytes T. Nous avons montré que ZAP-70 est nécessaire à la dégradation du TCR induite par sa stimulation [20].

La mort cellulaire induite par activation fait également partie des mécanismes inhibiteurs de la réponse T. Elle permet en particulier la délétion des clones autoréactifs en périphérie ou, au cours de l'onto-

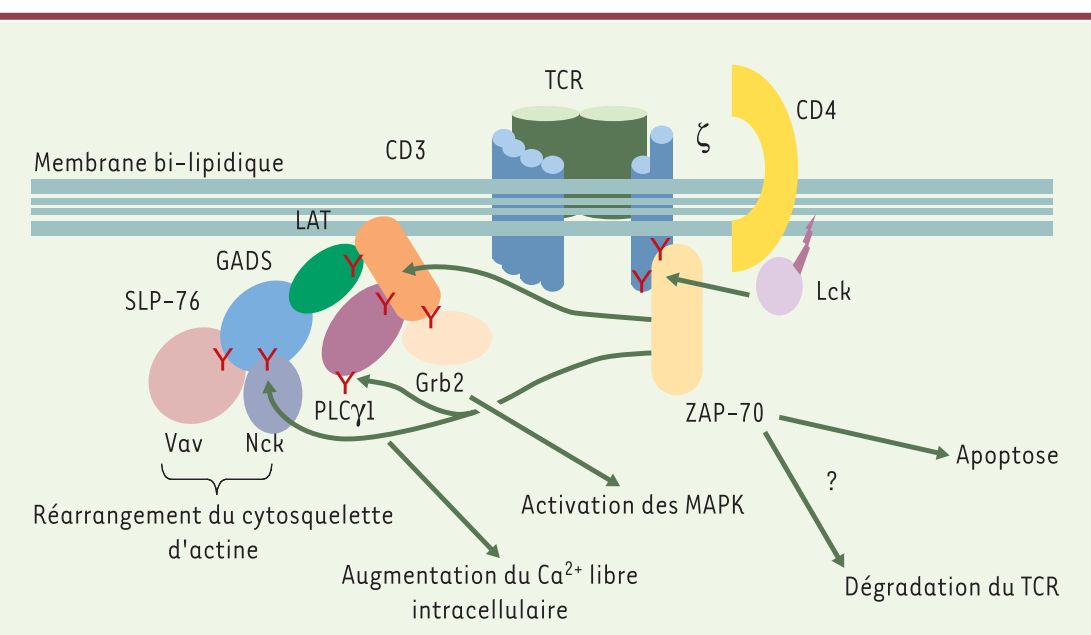

Figure 3. Voies de signalisation contrôlées par ZAP-70. Après stimulation du TCR, la tyrosine kinase p56 lck (lymphoid cell kinase), associée à la face interne de la membrane, phosphoryle des résidus tyrosine $(Y)$ présents dans les molécules $C D 3$ et $\zeta$. Cette phosphorylation permet le recrutement de ZAP-70, qui est alors phosphorylée par p56 $6^{\text {Lck }}$ et activée. ZAP-70 phosphoryle à son tour LAT sur plusieurs tyrosines. Une fois phophorylées, ces tyrosines servent d'ancrage à d'autres protéines de signalisation qui sont elles-mêmes phosphorylées et activées, permettant le remaniement du cytosquelette d'actine, un signal calcique et l'activation des MAPK. La fin de la réponse T est également contrôlée par ZAP-70, qui est nécessaire à l'apoptose et à la dégradation du TCR induites par stimulation de ce récepteur. LAT: linker for activation of T cells; SLP76: SH2-containing leukocyte protein of 76 kDa; GADS: Grb2-related adapter downstream of Shc; Grb2: growth factor receptor bound protein 2; PLC $\gamma 1$ : phospholipase C; MAPK: mitogenactivated protein kinase. génie, leur délétion dans le thymus. Cette mort cellulaire particulière, appelée apoptose, nécessite l'activité de ZAP-70 [21].

Enfin, il a récemment été montré que les protéines Stsl et Sts2, qui contrôlent l'activité de ZAP-70 [22], règlent négativement l'activation $T$. Les lymphocytes $T$ des souris n'exprimant ni Stsl, ni Sts2 présentent un état d'hyperactivation qui se traduit par une hyperphosphorylation des protéines ZAP-70, LAT et SLP76, ainsi qu'une production acrue de cytokines. Les mécanismes moléculaires qui sous-tendent cette inhibition, encore inconnus, semblent liés au contrôle de I'ubiquitinylation de ZAP-70. Les souris dépourvues de Stsl et Sts2 sont également plus sensibles à l'induction d'une maladie auto-immune expérimentale, un modèle murin de sclérose en plaques [22].

\section{ZAP-70 et auto-immunité}

Récemment, un groupe japonais a mis en évidence une mutation ponctuelle spontanée du gène murin zap-70 chez les souris SKG présentant une arthrite rhumatoïde très comparable à la polyarthrite rhumatoïde humaine. Chez ces animaux, et contrairement à ce qui est observé chez les souris zap-70 $70^{-/}$(voir schéma du rôle de ZAP-70 dans la différenciation des thymocytes, Figure 2), les lymphocytes $T$ $\mathrm{CD}^{+}$et $\mathrm{CD} 8^{+}$sont présents en périphérie [23]; en effet, la mutation dans le domaine $\mathrm{SH} 2(\mathrm{~N})$ (Figure I) affecte non pas l'expression de ZAP-70, mais son association avec le TCR, perturbant en aval la signalisation par ce récepteur. Chez ces souris, les sélections positive et négative des lymphocytes $T$ ne se font pas correctement. Celles-ci nécessitent en effet un niveau donné de signalisation par le TCR qui n'est pas atteint dans les thymocytes des souris mutantes. Les lymphocytes $T$ sélectionnés seront ceux qui présentent la plus forte avidité (et donc signalisation) pour les ligands qu'ils rencontrent lors de leur sélection: des peptides du soi associés aux molécules du complexe majeur d'histocompatibilité. Ainsi seront sélectionnés positivement des clones T autoréactifs qui, chez une souris « sauvage», auraient été sélectionnés négativement. 
De façon non exclusive, on peut imaginer qu'une activité ZAP-70 incorrecte empêche la délétion par apoptose des clones T autoréactifs qui, comme nous l'avons vu, est réglée par ZAP-70.

Ces résultats sont à rapprocher du phénotype observé chez un patient présentant un défaut partiel d'expression de ZAP-70 [8]. Chez ce patient, I'un des deux allèles de zap-70 code pour une protéine qui s'associe mal au TCR (mutation dans le domaine SH2) (Figure 1): cette mutation permet peut-être aux cellules d'acquérir un niveau d'activation suffisant pour induire une maturation des lymphocytes $T$, mais pas pour que la sélection positive et négative des thymocytes s'effectue correctement. Cela se traduit, comme chez la souris décrite ci-dessus, par la présence d'une population T autoréactive [9].

\section{ZAP-70 dans les lymphocytes B}

Contrairement à sa « sœur » Syk, dont l'expression dans les cellules hématopoïétiques est ubiquitaire, ZAP-70 a, dans un premier temps, été décrite comme une kinase spécifique des lymphocytes $T$ et des cellules NK. Cependant, un groupe américain a montré, en utilisant des puces à ADN, que certaines cellules de leucémie lymphoïde chronique $B(L L C B)$ exprimaient ZAP-70 [24]. Cette expression fut rapidement corrélée à un mauvais pronostic des LLC B, dont le diagnostic était auparavant établi en étudiant, par des méthodes complexes et coûteuses, le statut mutationnel du gène $\lg V H$ [25].

Outre son intérêt clinique, qui permettait d'utiliser des techniques plus simples pour réaliser un diagnostic pronostique, cette découverte suggérait que le récepteur des cellules $B(B C R)$ pouvait, comme le TCR, utiliser ZAP-70 pour induire une signalisation. La démonstration d'une phosphory- lation de ZAP-70 après stimulation du BCR est venue confirmer cette hypothèse [26]. L'hypothèse selon laquelle l'expression de ZAP-70 pourrait rendre les LLC B plus agressives en augmentant les capacités activatrices du BCR doit cependant être confirmée.

L'expression de Syk par les lymphocytes B et son rôle dans le développement et l'activation des lymphocytes $B$ ont été démontrés il y a 10 ans $[27,28]$. Cependant, plusieurs auteurs ont noté que le développement $B$ n'était que partiellement bloqué chez des souris déficientes en Syk [27, 28]. Ce bloquage seulement partiel pouvait être expliqué par la présence d'une autre kinase ayant des effets partiellement redondants; or, ZAP-70 est exprimée dans les lymphocytes B normaux de souris tout au long de leur différenciation [29]. Les souris n'exprimant ni Syk ni ZAP-70 présentent un blocage complet du développement des lymphocytes B. L'absence de Syk seule n'a pas d'effet aussi drastique, tandis que l'absence de ZAP-70 n'a aucun effet sur le développement B [29].

Le blocage complet de la différenciation $B$, chez les souris dont les gènes codant pour Syk et ZAP-70 ont été invalidés, démontre l'existence d'une redondance fonctionnelle entre ces deux protéines dans les lymphocytes B. Chez la souris, cette redondance fonctionnelle ne peut pas s'appliquer à la différenciation $T$, puisque les thymocytes murins n'expriment pas Syk [11]. En revanche, la sortie des lymphocytes $\mathrm{TCD}^{+}$humains en

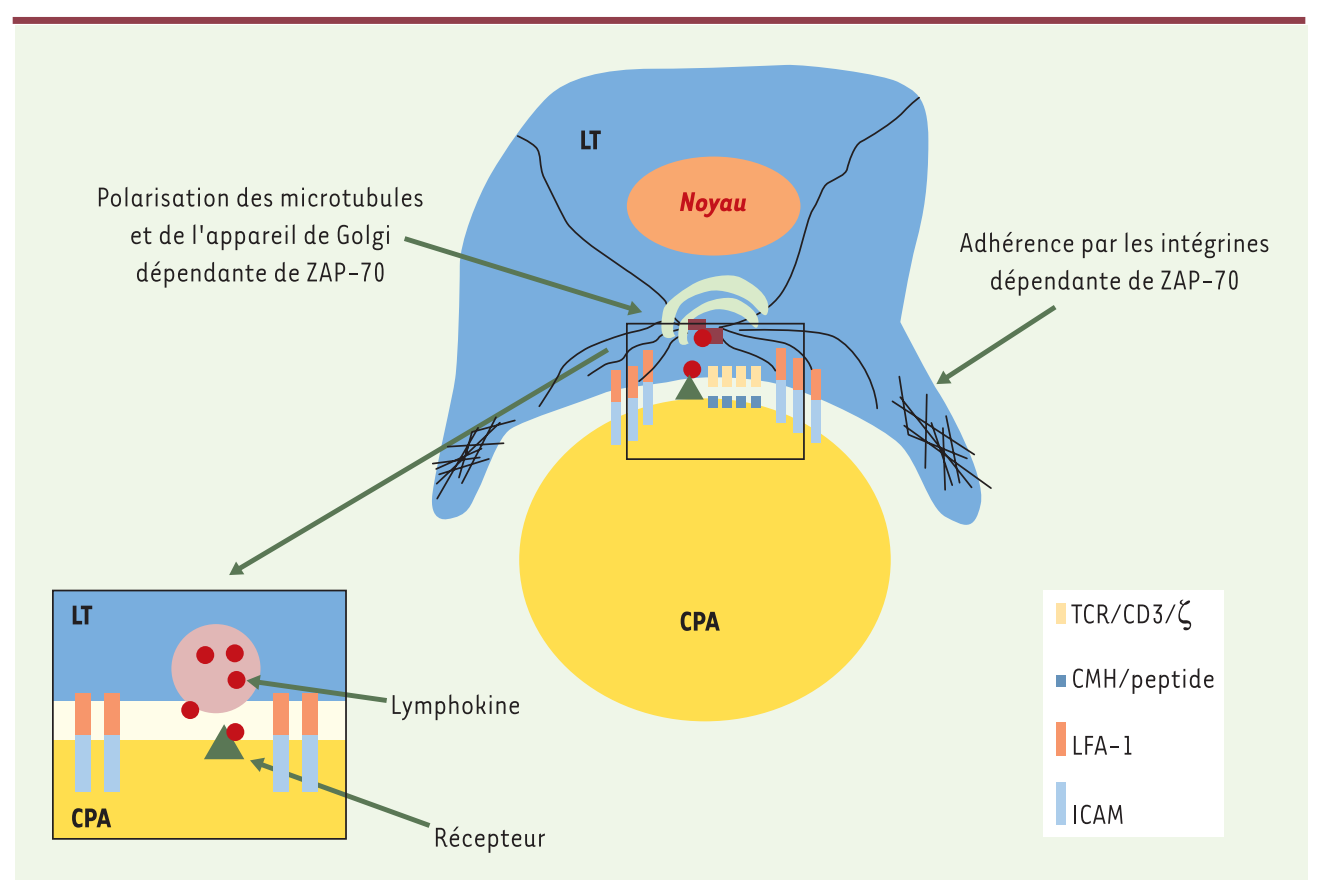

Figure 4. Contrôle de l'interaction lymphocyte T/cellule présentatrice d'antigènes par ZAP-70. Lorsqu'un lymphocyte T (LT) interagit avec une cellule présentatrice d'antigènes (CPA) qui porte l'antigène pour lequel il est spécifique, le lymphocyte T subit des remaniements améliorant le contact avec la CPA. Ces remaniements se traduisent notamment par une augmentation des capacités d'adhérence des intégrines et une polarisation des microtubules et de l'appareil de Golgi vers la CPA. Ces deux événements dépendent de l'activité de ZAP-70. L'encart montre comment l'adhérence et la polarisation de la sécrétion peuvent créer un lieu de concentration des lymphokines et éviter des effets non spécifiques sur des cellules avoisinantes. LFA-1: leukocyte function-adhesion molecule 1; ICAM: intercellular cell-adhesion molecule. 
périphérie pourrait être due à la présence de Syk dans les thymocytes humains [30]. La perte d'expression de Syk dans les lymphocytes $\mathrm{T} \mathrm{CD4}{ }^{+}$périphériques explique leur absence d'activité (Figure 2).

\section{Conclusions}

Le rôle de ZAP-70 dans la mise en place et la régulation d'une réponse immune est remarquablement illustré par les maladies associées à des défauts d'expression ou d'activité de cette protéine tyrosine kinase. ZAP-70 règle la signalisation par les immunorécepteurs, non seulement en permettant son «allumage », mais également en contrôlant son intensité et sa durée. Sur le plan fondamental, l'étude de cette kinase et de ses substrats devrait permettre de mieux comprendre les étapes clés de la régulation d'une réponse immune. Cette protéine fait également l'objet d'une recherche pharmacologique importante, constituant en effet une cible de choix dans le traitement des maladies auto-immunes ou dans l'induction d'une immunosuppression nécessaire aux greffes d'organes. $\diamond$

\section{REMERCIEMENTS}

Je tiens à remercier Nicolas Blanchard et Céline Dumont qui ont participé dans mon équipe aux travaux sur ZAP-70. Je souhaite également dédier cet article à Christian Bonnerot, qui nous a quitté trop tôt.

\section{SUMMARY}

Everything you ever wanted to know about ZAP-70

The ZAP-70 tyrosine kinase has been described more than ten years ago. Its key role in thymocytes development and mature $T$ lymphocytes activation has been illustrated by the characterization of several human immunodeficiencies presenting with mutations in the zap-70 gene resulting in the absence of ZAP-70 expression. More recently, it has been shown that deregulation of ZAP-70 activity can induce autoimmune diseases. Finally, ZAP-70 expression has been shown in some $B$ chronic lymphocytic leukaemia and correlated with bad prognosis of the disease. The diversity of pathologies associated with deregulation of ZAP-70 demonstrates its key role in immune responses. Research aiming at deciphering the different signalling pathways regulated by ZAP-70 will not only shed some lights on these pathologies, but will also help finding new pharmacological tools, targeting ZAP70 , designed to induce immunosuppression or tolerance. $\Delta$

\section{RÉFÉRENCES}

1. Chan AC, Iwashima M, Turck CW, Weiss A. ZAP-70: a 70 kd protein-tyrosine kinase that associates with the TCR zeta chain. Cell 1992; 71: 649-62.

2. Weiss A, Littman DR. Signal transduction by lymphocyte antigen receptors. Cell $1994 ; 76: 263-74$
3. Arpaia $\varepsilon$, Shahar M, Dadi H, et al. Defective T cell receptor signaling and CD8* thymic selection in humans lacking zap-70 kinase. Cell 1994; 76: 947-58.

4. Elder ME, Lin D, Clever J, et al. Human severe combined immunodeficiency due to a defect in ZAP-70, a T cell tyrosine kinase. Science 1994; 264 : 1596-9.

5. Chan AC, Kadlecek TA, Elder ME, et al. ZAP-70 deficiency in an autosomal recessive form of severe combined immunodeficiency. Science 1994; 264: 1599-601.

6. Hivroz C, Fischer A. Immunodeficiency diseases. Multiple roles for ZAP-70. Curr Biol $1994 ; 4: 731-3$.

7. Steinberg M, Swainson L, Schwarz K, et al. Retrovirus-mediated transduction of primary ZAP-70-deficient human T cells results in the selective growth advantage of gene-corrected cells: implications for gene therapy. Gene Ther 2000; 7: $1392-40$.

8. Matsuda S, Suzuki-Fujimoto T, Minowa A, et al. Temperature-sensitive ZAP70 mutants degrading through a proteasome-independent pathway. Restoration of a kinase domain mutant by Cdc37. J Biol Chem 1999; 274: 34515-8.

9. Katamura K, Tai G, Tachibana T, et al. Existence of activated and memory CD4 ${ }^{+} T$ cells in peripheral blood and their skin infiltration in CD8 deficiency. Clin Exp Immunol 1999; 115: 124-30.

10. Williams BL, Schreiber KL, Zhang W, et al. Genetic evidence for differential coupling of Syk family kinases to the T-cell receptor: reconstitution studies in a ZAP-70deficient Jurkat T-cell line. Mol Cell Biol 1998; 18: 1388-99.

11. Kadlecek TA, van Oers NS, Lefrancois L, et al. Differential requirements for ZAP-70 in TCR signaling and T cell development. J Immunol 1998; 161: 4688-94.

12. Meinl $\varepsilon$, Lengenfelder $D$, Blank $N$, et al. Differential requirement of ZAP-70 for CD2-mediated activation pathways of mature human T cells. J Immunol 2000; 165: 3578-83.

13. Shan X, Balakir R, Criado G, et al. Zap-70-independent $\mathrm{Ca}^{2+}$ mobilization and Erk activation in Jurkat T cells in response to T-cell antigen receptor ligation. Mol Cell Biol 2001; 21: 7137-49.

14. Zhang W, Sloan-Lancaster J, Kitchen J, et al. LAT: the ZAP-70 tyrosine kinase substrate that links T cell receptor to cellular activation. Cell 1998; 92: 83-92.

15. Wardenburg JB, Fu C, Jackman JK, et al. Phosphorylation of SLP-76 by the ZAP-70 protein-tyrosine kinase is required for T-cell receptor function. J Biol Chem 1996; 271: 19641-4.

16. Jordan MS, Singer AL, Koretzky GA. Adaptors as central mediators of signal transduction in immune cells. Nat Immunol 2003; 4: 110-6.

17. Goda S, Quale AC, Woods ML, et al. Control of TCR-mediated activation of beta 1 integrins by the ZAP-70 tyrosine kinase interdomain $B$ region and the linker for activation of T cells adapter protein. J Immunol 2004; 172: 5379-87.

18. Blanchard N, Di Bartolo V, Hivroz C. In the immune synapse, ZAP-70 controls T cell polarization and recruitment of signaling proteins but not formation of the synaptic pattern. Immunity 2002; 17: 389-99.

19. van Der Merwe PA, Davis SJ. The immunological synapse: a multitasking system. Science 2002; 295: 1479-80.

20. Dumont C, Blanchard N, Di Bartolo V, et al. TCR/CD3 down-modulation and zeta degradation are regulated by ZAP-70. J Immunol 2002; 169: 1705-12.

21. Zhong L, Wu CH, Lee WH, Liu CP. Zeta-associated protein of $70 \mathrm{kDa}($ ZAP-70), but not Syk, tyrosine kinase can mediate apoptosis of T cells through the Fas/Fas ligand, caspase- 8 and caspase- 3 pathways. J Immunol 2004; 172: 1472-82.

22. Carpino N, Turner S, Mekala D, et al. Regulation of ZAP-70 activation and TCR signaling by two related proteins, Sts-1 and Sts-2. Immunity 2004; $20: 37-46$.

23. Sakaguchi N, Takahashi T, Hata H, et al. Altered thymic T-cell selection due to a mutation of the ZAP-70 gene causes autoimmune arthritis in mice. Nature 2003; 426: 454-60.

24. Rosenwald A, Alizadeh AA, Widhopf G, et al. Relation of gene expression phenotype to immunoglobulin mutation genotype in B cell chronic lymphocytic leukemia. J Exp Med 2001; 194: 1639-47.

25. Orchard JA, Ibbotson RE, Davis Z, et al. ZAP-70 expression and prognosis in chronic lymphocytic leukaemia. Lancet 2004; 363: 105-11.

26. Chen L, Widhopf G, Huynh L, et al. Expression of ZAP-70 is associated with increased $B$-cell receptor signaling in chronic lymphocytic leukemia. Blood 2002; $100: 4609-14$

27. Turner M, Mee PJ, Costello PS, et al. Perinatal lethality and blocked B-cell development in mice lacking the tyrosine kinase Syk. Nature 1995; 378: 298-302.

28. Cheng AM, Rowley B, Pao W, et al. Syk tyrosine kinase required for mouse viability and B-cell development. Nature 1995; 378: 303-6.

29. Schweighoffer $\varepsilon$, Vanes L, Mathiot A, et al. Unexpected requirement for ZAP-70 in pre-B cell development and allelic exclusion. Immunity 2003; 18: 523-33.

30. Elder ME, Skoda-Smith S, Kadlecek TA, et al. Distinct T cell developmental consequences in humans and mice expressing identical mutations in the DLAARN motif of ZAP-70. J Immunol 2001; 166: 656-61. 